\title{
Carbon Fiber Laminates with Interlaminar Carbon Nanotubes
}

\author{
LEANDRO IORIO ${ }^{1}$, LOREDANA SANTO ${ }^{1}$, FABRIZIO QUADRIN ${ }^{1}$, DENISE BELLISARIO ${ }^{1}$, \\ DAVID BENEDETTI ${ }^{2}$, JACOPO AGNELLI ${ }^{2}$ \\ ${ }^{1}$ University of Rome "Tor Vergata", Department of Industrial Engineering, Via del Politecnico 1, Rome 00133, Italy \\ ${ }^{2}$ Carbon Dream SpA, Via Fausto Melotti 16, Tavarnelle Val di Pesa (FI), Florence, Italy
}

\begin{abstract}
Carbon fibre reinforced (CFR) laminates were manufactured by prepreg lay-up and deposition of interlaminar carbon nanotubes (CNTs). An easy and innovative manufacturing procedure was used. CNTs were separated in solvent by ultrasonication, and poured on the woven fabric prepreg. Solvent evacuation was performed at low temperature, and dry functionalized prepregs were used for composite lamination. Laminates were cured by compression moulding on a heating plate. Peeling tests, differential scanning calorimetry (DSC), and dynamic mechanical analyses (DMA) were carried out on multiply samples with and without $1 \mathrm{wt} \%$ of interlaminar CNTs. Results show that the glass transition temperature of the resin matrix reduces because of the interaction with CNTs. Nevertheless, peeling strength shows $10 \%$ increase at room temperature.
\end{abstract}

Keywords: Composite, Laminate, Carbon fibre, CNT, Manufacturing

\section{Introduction}

Carbon fibre reinforced (CFR) polymer laminates provide structural strength and stiffness for most of the applications of composite materials in aeronautics, racing and other high-added-value industrial fields. Improving manufacturing technologies and materials is a continuous need of industries to produce slighter and more reliable structures. Stiffness and in-plane strength mainly depend on the reinforcing fibres whereas other fundamental properties are influenced by the resin matrix such as interlaminar shear strength, creep, and peeling strength. By improving performances of the resin matrix, it is possible to improve also those properties but attention has to be paid to not affect resin processability. An example is given by the use of carbon nanotubes (CNTs) which can increase matrix strength and stiffness with very low amounts. However, as CNTs also increase liquid matrix viscosity and resin reactivity, they strongly affect composite manufacturing as well. The goal of improving CFR laminate performances by using CNTs is object of many recent scientific studies. The first step is defining a manufacturing technology which is able to provide the expected matrix strengthening to the laminate.

Dealing with autoclave or press moulding of thermosetting CFR composites, the interlaminar deposition of CNTs seems to be a valid solution but some scientific contributions also refer to the use massive mixing of CNTs in the resin matrix. In 2017, Leao et al. evaluated the positive effect of CNTs and multi-layer graphene in carbon/epoxy composites by tensile tests. Laminates were made by wet lamination of carbon fabrics with the filled uncured matrix [1]. They found that the ultimate stress increased around $18 \%$, while toughness had an improvement close to $62 \%$. Zhou et al. proposed to use hierarchical short carbon fibres synthesized with carbon nanotubes as interleaves to increase up to $125 \%$ the fracture energy of carbon fibre/epoxy (CF/EP) composite laminates [2]. The concept of interlaminar functional reinforcements was also developed by Zheng et al. which used vacuum filtration method to fabricate sandwiched CNT/polysulfone nanofiber paper as interleaf of the CFR laminates [3]. They found that laminates with the sandwiched paper increased both mode I and mode II interlaminar toughness. Analogously, Kaynan et al. developed micron-scale thin carbon nanotubes reinforced adhesive nano-fibrous interleaves, and used to produce interlayered CFR laminates [4]. Despite of resulting performances, manufacturing of interleaves in the shape of hierarchical fibres, papers or adhesive layers is not an easy step.

*email: leandro.iorio@uniroma2.it 
In 2018, Mikhalchan et al. discussed that CNT assemblies such as vertical arrays and mats have the potential to provide additional interlaminar toughness for carbon fibre-reinforced laminates but they concluded that the achieved improvement in mechanical properties was not always been consistent so far [5]. In their technological proposal, they introduced thin interlayers of continuous unidirectional CNT fibres for additional in-plane reinforcement and toughening of conventional autoclave-cured carbon fibre composites. Tensile tests on double-notched specimens with and without CNT fibre reinforcing layers showed that the introduction of relatively thin interlayers of continuous CNT fibres at local regions close to the notch tip increased the failure load and stress by $9 \%$ and the failure strain by $15 \%$ respectively, suggesting the toughening potential of CNT fibres. Nevertheless, the processing solution seems to be complex because of the need of winding 80 - $100 \mathrm{~m}$ of CNT fibres on a metal mandrel. An alternative could be spreading CNTs in the full matrix resin and not only in the interlaminar region. Abdelal et al. evaluated vacuum bagging process to prepare carbon fibre/CNT composites but they found negligible improvements of the strength which were covered by data scattering [6]. In the same year, Yourdkhani et al. started with the consideration that effective dispersion of CNTs in the polymer matrix of fibrereinforced composites is challenging due to the re-agglomeration and filtration of CNTs that occur during liquid processing [7]. They used resin film infusion process for manufacturing composite laminates and investigating the degradation of CNT dispersion during high-temperature processing. Two lay-up strategies were selected, grouped and interleaved, and results showed that laminates manufactured by interleaved lay-up exhibited higher compressive strength and electrical conductivity even if the comparison with neat laminates was not impressive. However, they confirmed that interlaminar manufacturing strategies seem to be more promising.

More recently, in the current year, Li et al. have proposed the use of CNT buckypaper to the midplane of glass fibre reinforced laminates [8]. In all the recent achievements, the insertion of CNTs is performed by placing functional layers which need a complex manufacturing procedure, and the modification of the laminate stacking sequence. In this study, a new technological solution is proposed to add interlaminar CNTs without any additional manufacturing step of intermediate interleaves. CNTs are deposited on the surface of commercial CFR prepregs before lamination by solvent mixing. After solvent evacuation, the composite is laminated and cured. The lamination technique has been prototyped at lab-scale to produce interlaminar carbon nanotubes (I-CNT) CFR laminates. Differential scanning calorimetry (DSC) and dynamic mechanical analysis (DMA) were used to study the interaction between CNTs and composite matrix. As a proof of concept, mechanical testing by peeling was carried out, and promising results have been obtained and are discussed in the following.

\section{Materials and methods}

Composite laminates for mechanical testing were made in laboratory by compression moulding of commercial epoxy matrix prepregs ( $0 / 90$ fabrics by Microtex Composites Srl, Prato, Italy). Burning tests revealed that $\mathrm{CF}$ content was $50 \mathrm{wt} \%$. Commercial multi-walled carbon nanotubes (MWCNTs, 30-50 $\mathrm{nm}$ ) were acquired and used as interlaminar reinforcement. Fig.1 shows the proposed manufacturing technology. MWCNTs were diluted into isopropanol and sonicated for $40 \mathrm{~min}$. This step is fundamental to achieve a sufficient level of MWCNT separation before their deposition. The effect of sonication is also evident at naked eye as MWCNT agglomerates disappear, and a black continuous solution is made (Fig.2a). Subsequently the sonicated solution was poured on prepreg sheets. As MWCNTs only interact with the resin matrix, their filling content was referred to that, and not to the overall composite. In particular, the MWCNT content was fixed to $1 \mathrm{wt} \%$ of the resin content of the single CFR prepreg ply.

Functionalized prepreg sheets were placed in fridge at $4^{\circ} \mathrm{C}$ for solvent evacuation. The following step was cutting the sheets in the desired shape for the final samples, $10 \times 50 \mathrm{~mm}^{2}$ for peeling tests, $10 \times 60$ $\mathrm{mm}^{2}$ for DMA analyses. Lamination was made by putting in contact the top functionalized surface of a CFR strip with the bottom non-functionalized surface of the further strip. The last strip was not functionalized. For DMA testing, 2-ply laminates were used whereas multiply laminates were manufactured for peeling. Moreover, in the case of peeling tests, samples were moulded with double-T 
shapes according to Fig. $2 \mathrm{~b}$, and MWCNTs were inserted only in the middle plane. Laminates were cured on a heater plate at $150^{\circ} \mathrm{C}$ by applying a small pressure $(10 \mathrm{kPa})$ for $30 \mathrm{~min}$. Samples with and without MWCNTs were produced.

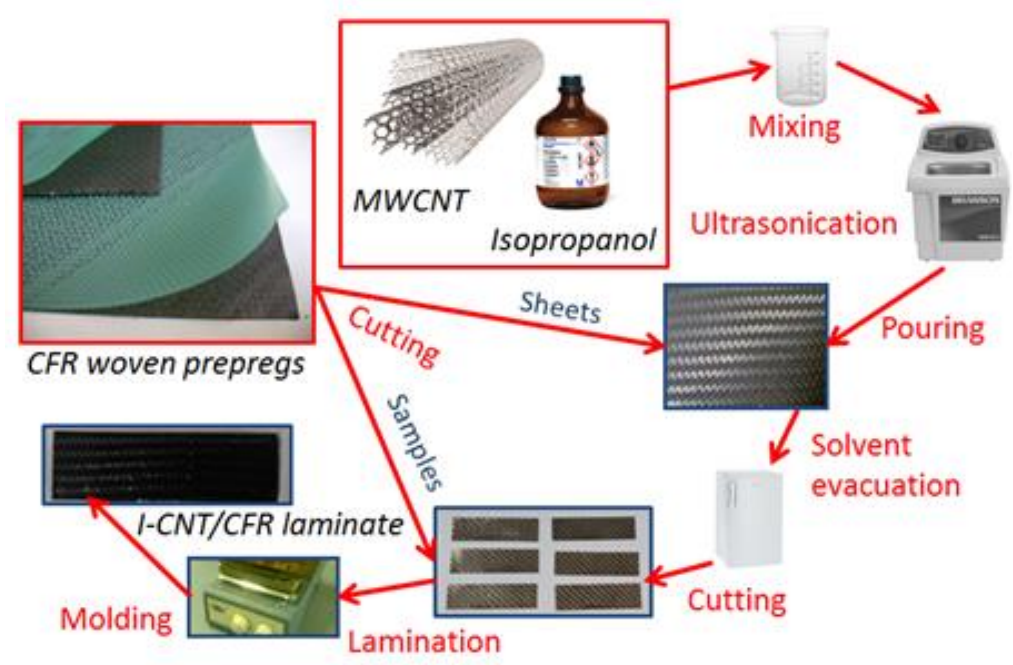

Figure 1. Manufacturing procedure for I-CNT/CFR samples

a)

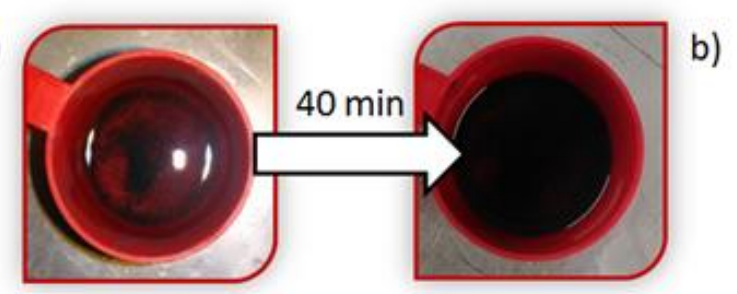

b)

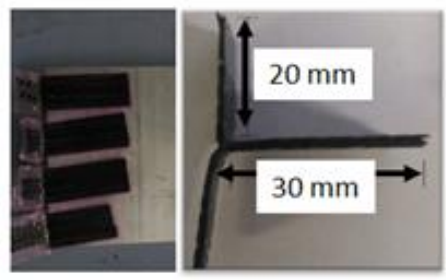

Figure 2. Manufacturing procedure for I-CNT/CFR samples

Peeling tests were performed on a universal material testing machine (Alliance Insight 5 by MTS) by clamping sample ends with tensile grips and applying the rate of $1 \mathrm{~mm} / \mathrm{min}$. DMA (by Netzsch DMA 242C) and DSC (by Perkin Elmer DSC6) tests were performed on 2-ply laminates with and without interlaminar CNTs. DMA tests were carried out in the bending configuration by temperature scans from room temperature to $200^{\circ} \mathrm{C}$ at the heating rate of $5^{\circ} \mathrm{C} / \mathrm{min}$, the frequency of $10 \mathrm{~Hz}$, and the span-length of $40 \mathrm{~mm}$. DSC tests were carried out from $0^{\circ} \mathrm{C}$ to $230^{\circ} \mathrm{C}$ at $10^{\circ} \mathrm{C} / \mathrm{min}$. Double scans were made starting from uncured samples for DSC and already cured samples for DMA.

\section{Results and discussions}

The positive effect of CNTs on the mechanical properties of composite laminates depends on the reduction of molecular mobility because of the interaction between polymer matrix and nano-particles. This effect is particularly strong if interpenetrating structures are produced in the interlaminar region. However, in liquid processing of thermosetting resins molecular mobility is important. In fact, low viscosity is necessary for fibre impregnation and high diffusion for high polymerization degrees. Both viscosity and diffusion are strongly limited by the same CNTs. Therefore, in order to correctly design the integration of CNTs in polymeric matrix composites it is necessary to balance advantages and disadvantages. CNTs are useful in the final thermoset matrix but they affect prepreg and laminate processing. For this reason, new manufacturing solutions are always welcome to move this equilibrium toward higher advantages. 


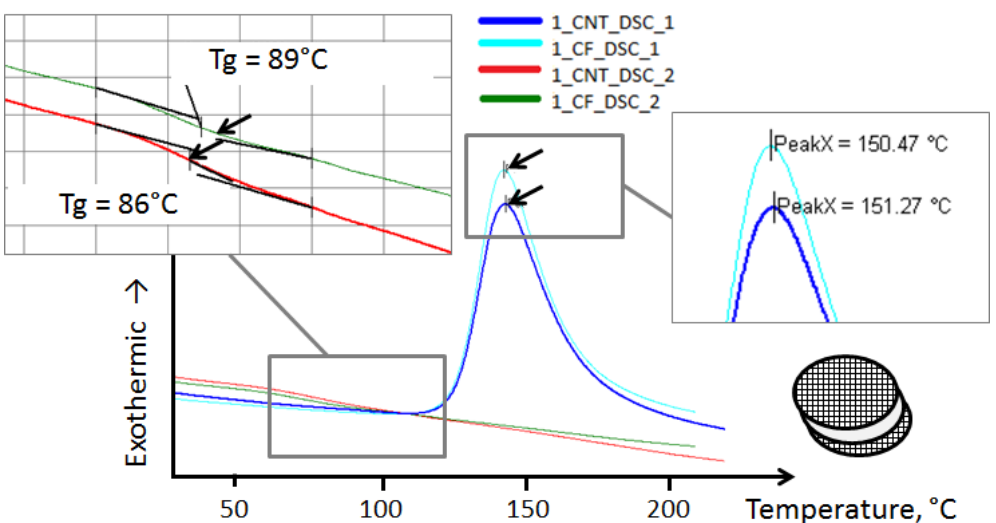

Figure 3. DSC double scans of neat (CF_DSC) and CNT functionalized (CNT_DSC) composite laminates
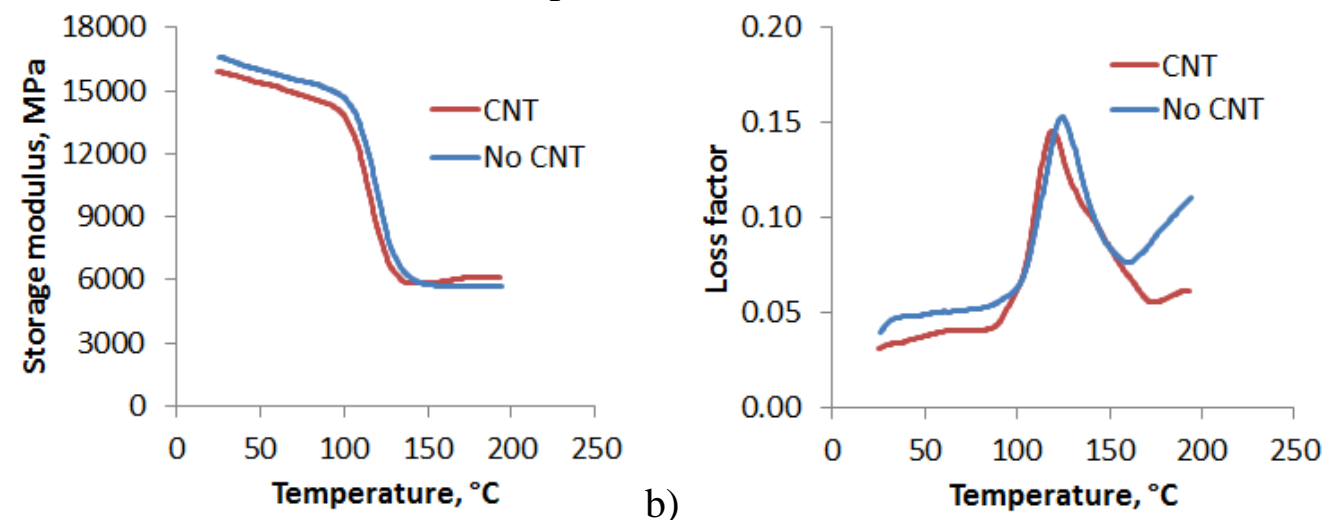

Figure 4. Comparison between storage modulus (a) and loss factor (b) for neat and CNT functionalized laminates (second scans)

The proposed manufacturing method is the first one which is able to add interlaminar CNTs without adding an additional layer to the composite laminate in the shape of adhesives or interleaves. Commercial prepregs are functionalized without modifying the laminate resin content by means of a simple technique which can be easily transferred also to an industrial environment. The only technological step which is added to traditional composite lamination is related to the sonication of an isopropanol solution with CNTs inside. Scientific literature has shown that interlaminar CNTs provide high performances to functionalized laminates if the manufacturing process is correctly set-up.

Results from DSC double scans are shown in Fig.3. In the first scan, an exothermic peak is visible for both samples because of the prepreg resin cure. In the second scan, reaction peaks disappear and glass transition regions of the cured samples are visible. Because of the high fibre content, the glass transition temperature is hard to estimate but a first comparison is possible. The reaction peak of the composite laminate is higher than the reaction peak of the SMP composite and, as a consequence, a bigger reaction heat is achieved. Due to this difference, a lower glass transition temperature is observed in the second scan for the I-CNT/CFR composite. Glass transition temperature reduces from $89^{\circ} \mathrm{C}$ to $86^{\circ} \mathrm{C}$. The main result from DSC tests is that an interaction between the resin matrix and the CNTs has been achieved with the proposed manufacturing method also for the low size sample. This interaction has been confirmed by DMA data where only cured samples were tested.

Table 1. Glass transition temperature from DMA analyses

\begin{tabular}{|c|c|c|c|c|}
\hline \multirow{3}{*}{$\mathbf{T g}\left({ }^{\circ} \mathbf{C}\right)$} & \multicolumn{2}{|c|}{$\mathbf{E}^{\prime}$ inflection } & \multicolumn{2}{c|}{ tgo peak } \\
\hline \multirow{3}{*}{ Neat } & First scan & Second scan & First scan & Second scan \\
\cline { 2 - 5 } & 116.2 & 121.3 & 122.2 & 124.3 \\
\cline { 2 - 5 } & 113.3 & 115.9 & 121.2 & 122.5 \\
\cline { 2 - 5 } & 112.5 & 119.1 & 124.7 & 127.3 \\
\hline
\end{tabular}




\begin{tabular}{|c|c|c|c|c|}
\hline & 114.0 & 118.8 & 122.7 & 124.7 \\
\hline \multirow{3}{*}{ I-CNT } & 108.0 & 115.0 & 117.4 & 119.2 \\
\cline { 2 - 5 } & 103.1 & 114.0 & 112.9 & 120.7 \\
\cline { 2 - 5 } & 108.3 & 105.3 & 114.0 & 122.2 \\
\cline { 2 - 5 } & 106.5 & 111.4 & 114.8 & 120.7 \\
\hline Relative difference & $-7.1 \%$ & $-6.6 \%$ & $-6.9 \%$ & $-3.3 \%$ \\
\hline
\end{tabular}

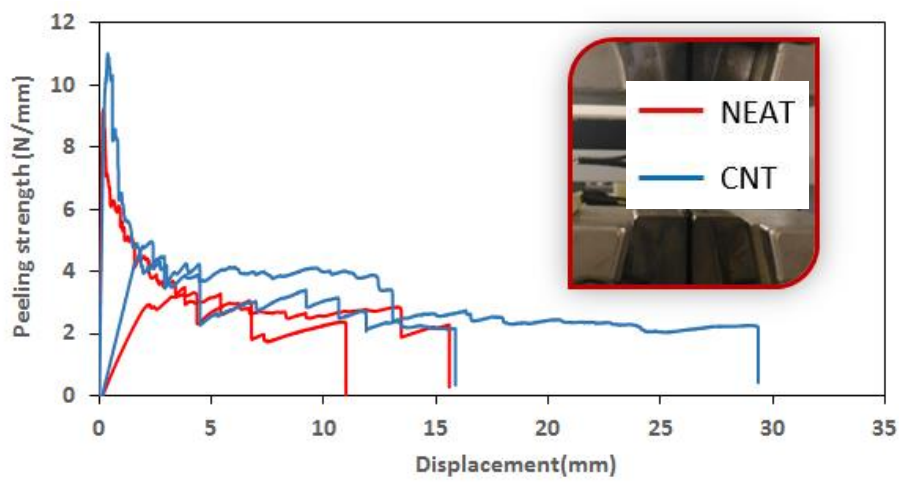

Figure 5. Peeling strength of neat and CNT functionalized laminates

DMA testing is better to evaluate the effect of the CNT integration because of the impact of CNTs on resin mobility. For DMA samples, a thickness of $0.47 \pm 0.05 \mathrm{~mm}$ and a density of $1.27 \pm 0.05 \mathrm{~g} / \mathrm{cm}^{3}$ were achieved for the neat laminate. The values for the I-CNT/CFR laminate were similar: 0.49 \pm 0.01 $\mathrm{mm}$ and $1.21 \pm 0.01 \mathrm{~g} / \mathrm{cm}^{3}$. Even if differences are small, functionalized samples were thicker and lighter probably due to the difficulty of resin flow during prepreg consolidation. That is another negative effect related to the presence of CNTs. In DMA, double scans were made to homogenize samples by providing stress release and post curing in the first scan: 3 tests were carried out for each laminate typology. Figure 4 shows a typical comparison between a sample with and without interlaminar CNTs in terms of storage modulus (E') and loss factors (tg $\delta$ ). Mechanical data are comparable but resin glass transition, which is related to the inflection point of the storage modulus or the peak of the loss factor, moves toward lower temperatures. A quantitative evaluation of the glass transition temperature $\mathrm{T}_{\mathrm{g}}$ is provided in Table 1 for all the samples. Values from $\operatorname{tg} \delta$ peaks are always higher than values from E' inflections. In the second scan, $T_{g}$ increases in both cases but values for neat laminates are always higher than values from ICNT/CFR laminates. DMA data confirm that an interaction has been achieved between resin and CNTs even if negative effects are measured.

The negative effects of the CNT interaction are observed at high temperatures but improvements could be obtained at room temperature because of the same mechanism of molecular mobility reduction. In the current work, peeling tests have been used for this aim. Actually, it was not easy to obtain reproducible results for I-CNT/CFR laminates because of some typical process variabilities which are difficult to take under control in laboratory. It is important that the laminate does not change its orientation during testing. In the end, it was found a good compromise by using 8-ply laminates for peeling tests. CNTs were only added in the middle layer where cracks propagate whereas the other interfaces were CNT free.

In Figure 5 the comparison between 2 tests on neat laminates and 2 tests on I-CNT/CFR laminates is shown. The curves show the normalized peeling load which is the peeling load divided by the width. The peeling strength may be extracted by the plateau values of Figure 5. Because of the un-homogeneous nature of the composite laminate, several oscillations occur but the effect of the CNT insertion is visible. In fact, the peeling curve of the I-CNT/CFR laminate is generally higher than the neat composite. The peeling strength resulted to be $2.60 \pm 0.05 \mathrm{~N} / \mathrm{mm}$ for the neat laminate and $2.87 \pm 0.06 \mathrm{~N} / \mathrm{mm}$ for the ICNT/CFR laminate with $10 \%$ increase. Even if this is a preliminary experimentation and many more tests and laminate configurations would be necessary, results from peeling tests show that it is possible 
to increase CFR laminate performances by using I-CNTs. Discussed increase in peeling strength is in agreement with expectations by literature reviews.

\section{Conclusions}

In the composite world, material innovation means improving already high material performances by using simple industrial processes. Using commercial materials seems to be the best solution at least for high value applications where material approval and supply undergo strong restrictions and quality controls. In the end, any laminate improvement should minimally affect the initial laminate structure and composition to avoid structure re-designing. The proposed manufacturing innovation is able to match all these necessities. It is easy to perform as it uses common solvents and does not affect the lamination procedure. Only commercial materials are involved in the composite manufacturing and there is no alteration of the composite structure apart from the addition of a small amount of CNTs. DSC and DMA tests show that the interaction between the resin matrix of the prepregs and the deposited CNTs has been achieved. Moreover, the peeling strength increases up to $10 \%$ in comparison with neat laminates. In the future, different laminate configurations and CNT contents will be tested for a fully evaluation of the potential of this method. Further analyses are also necessary to fully understand the delamination mechanism (adhesive/cohesive) and the distribution of CNTs at the interfaces. The optimal CNT content should be fixed as a function of the manufacturing procedure as aggregated bundles and damage of CNTs could affect composite mechanical properties. Other potential advantages of CNT on electrical properties or EM shielding could be investigated as well.

Acknowlegments: This article was presented at Polymer Processing in Engineering Conference - PPE 2019, 7- 9 October 2019, Galati - Romania.

\section{References}

1.LEÃO, S.G., MARTINS, M.G., MENEZES, N.C.F., LIMAM F.L.R.M., SILVA, C.F., ARANTES, G.C., ÁVILA, A.F. Mater Res, 20, 2017, p.134.

2.ZHOU, H., DU, X., LIU, H.Y., ZHOU, H., ZHANG, Y., MAI, Y.W. Compos Sci Technol 2017, 140, p.46.

3.ZHENG, N., HUANG, Y., LIU, H.Y., GAO, J., MAI, Y.W. Compos Sci Technol 2017, 140, p.8.

4.KAYNAN, O., ATESCAN, Y., OZDEN-YENIGUNA, E., CEBECI, H. Compos Part B, 2018, 154, p.194.

5.MIKHALCHAN, A., RIDHA, M., TAY, T.E. Mater Des 2018, 143, p.112.

6.ABDELAL, N.R., AL-SALEH, M.H., IRSHIDAT, M.R. Polym-Plast Technol, 2018, 57, p.175.

7.YOURDKHANI, M., LIU, W., BARIL-GOSSELIN, S., ROBITAILLE, F., HUBERT, P. Compos Sci Technol, 2018, 166, p.169.

8.LI, N., WANG, G.D., MELLY, S.K., PENG, T., LI, Y.C., ZHAO, Q.D., JI, S.D. Compos Struct, 2019, 208, p.13.

Manuscript received: 6.02.2020 